\title{
MONITORAMENTO FÍSICO-QUÍMICO E MICROBIOLÓGICO DE REATORES RBS TRATANDO ESGOTO BRUTO COM E SEM ADIÇÃO DE MICRO-ORGANISMOS EFICIENTES
}

\author{
D. H. NAKAGAWA ${ }^{1}$, C. Z. CORREA ${ }^{1}$, B. L. BABINSKI ${ }^{1}$, K. V. M. C. PRATES ${ }^{2}$ e B. O. FREITAS ${ }^{2}$ \\ ${ }^{1}$ Universidade Tecnologia Federal do Paraná \\ ${ }^{2}$ Universidade Tecnológica Federal do Paraná, Departamento de Engenharia Ambiental \\ E-mail para contato: daninakagawa@hotmail.com
}

RESUMO - O objetivo do trabalho foi avaliar a influência da adição de Micro-organismos Eficientes (EMs) no tratamento de esgoto sanitário, utilizando Reator em Bateladas Sequenciais (RBS) Aeróbio com biomassa suspensa e aderida. Realizou-se análises de DQO e série de sólidos ao longo de toda a operação de dois reatores, um com adição de EMs (RBSAe1) e outro sem (RBSAe2), e análises de quantificação de Bactérias Heterotróficas $(\mathrm{BH})$ e Fermentadoras de Lactose (BFL), Leveduras (LEV)e Actinomicetos (ACT) no meio suporte (espuma) e no lodo no $28^{\circ}$ e $76^{\circ}$ ciclo. A estabilização da remoção de matéria orgânica ocorreu no $25^{\circ}$ ciclo, com eficiência média de remoção de DQO Filtrada de $80 \%$. A remoção de ST, STV, SST e SSV foram respectivamente de 54\%, $87 \%$, $70 \%$ e $86 \%$ no RBSAe1 e de $56 \%, 86 \% 73 \%$ e $84 \%$ no RBSAe2. No ciclo 28 , após a estabilização dos reatores as $\mathrm{BH}, \mathrm{ACT}$, LEV e BFL presentes no lodo apresentaram valores, respectivamente, na ordem de $10^{7}, 10^{6}, 10^{5}$ e $10^{5} \mathrm{UFC} / \mathrm{mL}$ no RBSAe1 e $10^{7}, 10^{7}, 10^{5}$ e $10^{5} \mathrm{UFC} / \mathrm{mL}$ no RBSAe2 e na espuma foi de $10^{10}, 10^{8}, 10^{7}$ e $10^{6}$ UFC/espuma no RBSAe1 e $10^{10}, 10^{8}, 10^{7}$ e $10^{5}$ UFC/espuma no RBSAe2. Não houve diferença significativa na eficiência de remoção de DQO e sólidos entre os reatores, inferindo que a quantidade de EMs da solução utilizada neste estudo não foi o suficiente para prover diferença da população no RBSAe1, porém os EMs são capazes de se desenvolver em esgoto tornando estes um potencial agente biológico consumidor da matéria orgânica presente em esgoto.

\section{INTRODUÇÃO}

O trabalho com Micro-organismos Eficientes (EMs - do inglês "Efficient Microorganisms") foi iniciado pelo Professor Teruo Higa da Universidade de Ryukyus em Okinawa (Japão), com o objetivo de melhorar a utilização da matéria orgânica na produção agrícola (BONFIM et al., 2011). Os EMs produzem substâncias bioativas que controlam espécies patogênicas e favorecem a multiplicação de micro-organismos benéficos ao meio e sua aplicação tem aumentado a eficiência de remoção dos parâmetros como demanda bioquímica de oxigênio (DBO), sólidos em suspensão, odor e coliformes (BENERJI; WOOD; FARRELLY, 2002; BONFIM et al., 2011).

Os EMs consistem em uma mistura de micro-organismos que ocorrem normalmente no solo e plantas. Os micro-organismos de maior predominância no EMs são as bactérias fermentadoras de lactose (BFL) e leveduras (LEV), e de menor número os actinomicetos (ACT), as bactérias fotossintéticas e outros tipos de organismos. 


\section{9 a 22 de outubro de 2014 \\ Florianópolis/SC}

Estudos indicam que a utilização de EMs no tratamento de efluentes aumenta a eficiência do sistema, tornando a aplicação de EMs uma alternativa atrativa. No trabalho de Aguilar (2012) utilizando três tratamentos: T1: efluente de suinocultura, T2: efluente de suinocultura + EMs capturado pelos autores, T3: efluente de suinocultura + EMs comercial, com adição de 1/1000 (volume de EMs/volume de efluente), o autor observou que nos tratamentos com EMs houve maiores remoções de DBO, DQO e sólidos totais. Balasubramanian e Muthukumar (2012) utilizaram reator hibrido anaeróbio de fluxo ascendente com manta de lodo no tratamento de efluente industrial de papel celulose e aplicou uma concentração de 1/1000 de EMs por dia ao sistema e alcançou remoção de $92 \%$ de DQO.

Diante do exposto, o presente trabalho teve como objetivo avaliar a influência da adição de EMs em tratamento de esgoto sanitário, comparando a eficiência de dois Reatores Bateladas Sequenciais (RBS) Aeróbio com biomassa suspensa e aderida sendo um operado com adição de EMs e outro sem.

\section{MATERIAL E MÉTODOS}

\subsection{Descrição e operação dos reatores}

Os reatores utilizados no trabalho foram confeccionados em polipropileno, diâmetro de $24,6 \mathrm{~cm}$ e altura de $50 \mathrm{~cm}$, resultando em um volume total de aproximadamente $24 \mathrm{~L}$, sendo o volume útil de $10 \mathrm{~L}$ e o volume adicionado para tratamento de $6,5 \mathrm{~L}$. Cada reator possuía um tubo de descarte fixado no fundo, disposto a $6,2 \mathrm{~cm}$ da base, a fim de manter aproximadamente $20 \%$ do lodo.

O meio suporte utilizado para formação do biofilme, era composto por espuma de poliuretano de $2 \mathrm{~cm}$ de largura, 7,5 $\mathrm{cm}$ de comprimento e $1 \mathrm{~cm}$ de espessura (densidade de $15 \mathrm{Kg} / \mathrm{m}^{3}$ ). A espuma foi envolta por anéis de polipropileno perfurados, com $3 \mathrm{~cm}$ de diâmetro e $2 \mathrm{~cm}$ de altura. Seguindo as recomendações de Aygun et al. (2008), foram inseridos materiais suportes equivalente a $50 \%$ do volume útil.

Os reatores foram alimentados com esgoto bruto da ETE-Norte da cidade de Ibiporã-PR e os EMs adicionados no início do ciclo foram coletados conforme descrito no Caderno dos Microrganismos Eficientes (BONFIM et al., 2011). O local escolhido para a captura dos EMs foi uma propriedade rural a 5 quilômetros do distrito de Warta - PR, com acesso na rodovia PR-445 a $23^{\circ} 10^{\prime} 56^{\prime \prime} \mathrm{S}$ e $51^{\circ} 12^{\prime} 46^{\prime \prime} \mathrm{O}$.

Os reatores foram operados com ciclos de 8 horas, sendo um com adição de EMs no início de cada ciclo (RBSAe1) e outro sem adição de EMs (RBSAe2). A proporção de EMs adicionada no RSBAe1 foi de 1/1000 (EMs/esgoto), assim como nos estudos realizados por Maalin et al. (2009), Aguilar (2012) e Balasubramanian e Muthukumar (2012) e confirmada como a proporção mais satisfatória por Nurul et al. (2013).

Com o auxílio de uma bomba dosadora, no início de cada ciclo era adicionado no RBSAe1 6,5 $\mathrm{mL}$ de solução de EMs, juntamente com o esgoto bruto (etapa de alimentação). O monitoramento foi conduzido durante 76 ciclos de operação dos reatores, sendo que não houve adição de inoculo para a 
partida do reator. As características operacionais de cada ciclo estão descritas e representadas na Figura 1.

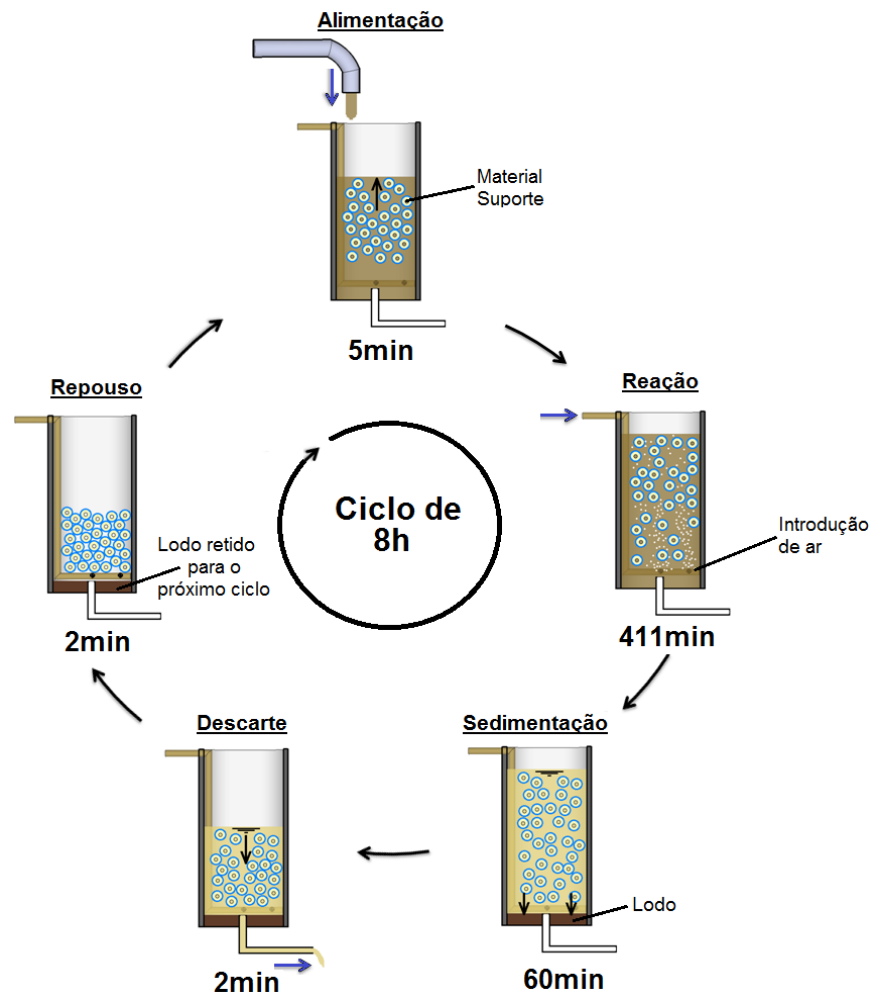

Figura 1 - Descrição do ciclo operacional dos reatores utilizados no experimento.

\subsection{Análises Físico-químicas}

Para avaliar o desempenho dos reatores foram realizadas as análises físico-químicas de Demanda Química de Oxigênio (DQO), Sólidos Totais (ST), Sólidos Totais Voláteis (STV), Sólidos Suspensos Totais (SST) e Sólidos Suspensos Voláteis (SSV), seguindo os métodos descritos no Standard Methods for Examination of Water and Wastewater (APHA; AWWA, WEF, 2012).

\subsection{Análises microbiológicas}

Foram avaliados a quantidade de micro-organismos na solução de EMs adicionada e no esgoto bruto. Os EMs avaliados foram as BFL, LEV e ACT, além de Bactérias Heterotróficas (BH). Os meios específicos para a contagem dos micro-organismos foram o meio Agar MRS $\left(36 \pm 1{ }^{\circ} \mathrm{C}\right)$ para BFL, meio Yeast Peptone Dextrose (YPD) $\left(30 \pm 1{ }^{\circ} \mathrm{C}\right)$ para leveduras, meio sólido Amido Caseína (AC) $\left(28 \pm 1^{\circ} \mathrm{C}\right)$ para ACT e meio Plate Count Agar $(\mathrm{PCA})\left(36 \pm 1^{\circ} \mathrm{C}\right)$ para $\mathrm{BH}$. 
Para a quantificação dos EMs na solução de EMs e no esgoto foi retirado uma alíquota de 10 $\mathrm{mL}$ da amostra e realizada a diluição seriada em solução salina a $0,85 \%$ até a diluição $10^{-5}$ e então espalhada $0,1 \mathrm{~mL}$ em placa de Petri contendo o meio específico para cada grupo a ser isolado. Após o tempo de incubação foi realizada a contagem das colônias características desenvolvidas e determinada a quantidade de Unidade Formadora de Colônia (UFC) por mL.

Foram escolhidos dois momentos para a quantificação de EMs e BH no meio suporte e no lodo, no $28^{\circ}$ ciclo (após a estabilização dos reatores) e no $76^{\circ}$ (final da operação dos reatores). Para quantificar a biomassa aderida, uma amostra do material suporte foi retirada do reator na fase de repouso. A espuma foi depositada em tubo Falcon $(50 \mathrm{~mL})$ estéril, contendo $10 \mathrm{~mL}$ de solução de Tween 80 a $1 \%$ e $5 \mathrm{~g}$ de pérolas de vidro. Após inserida a espuma, foi adicionada mais $5 \mathrm{~g}$ de pérolas de vidro e agitada em Vortex por 1 min para liberação de células bacterianas (Figura 2), do sobrenadante foram realizadas diluições seriadas e submetida as análises microbiológicas.

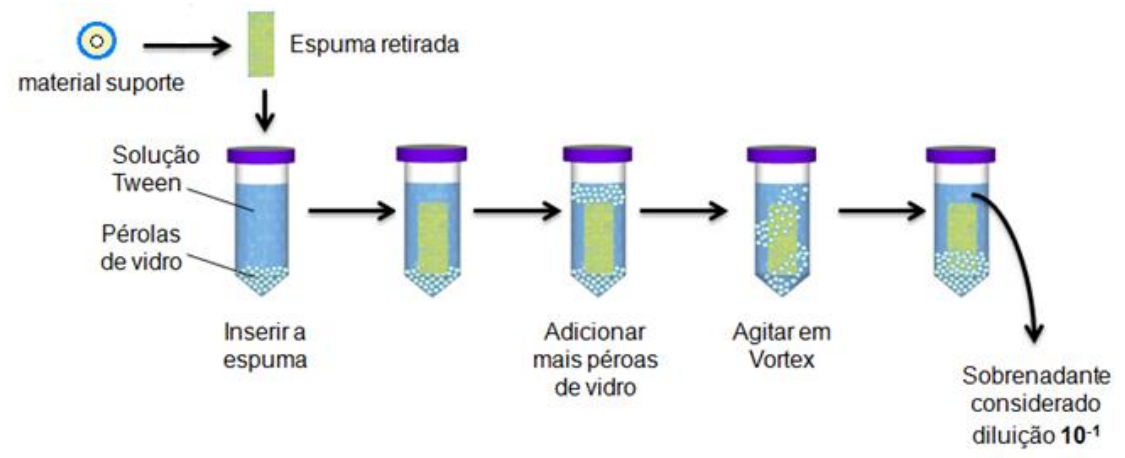

Figura 2 - Ilustração da metodologia para a obtenção da diluição $10^{-1}$ obtida do material suporte.

Uma alíquota de $1 \mathrm{~mL}$ de lodo foi retirada do reator na fase de repouso e transferido para um tubo contendo $9 \mathrm{~mL}$ de solução salina a $0,85 \%$ e homogeneizada (Figura 3), e deste foi realizado diluições seriadas e submetidas as análises microbiológicas.

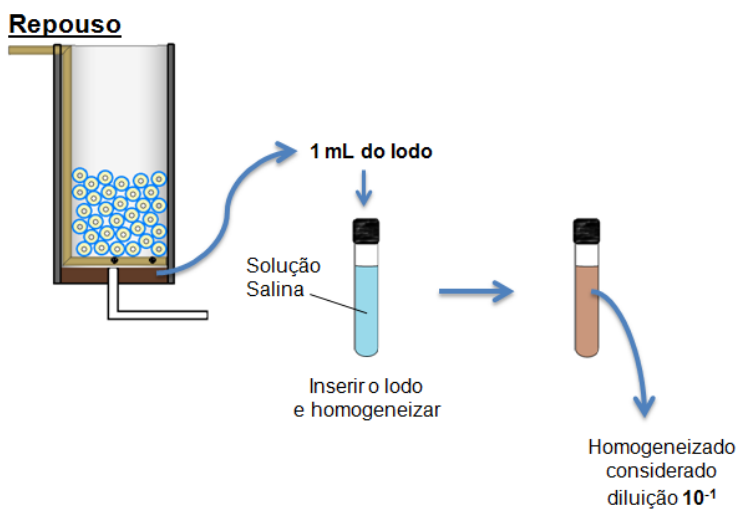

Figura 3 - Ilustração da metodologia para a obtenção da diluição $10^{-1}$ do lodo. 


\section{Resultados e discussões}

\subsection{Análises físico-químicas}

A partir das análises de $\mathrm{DQO}_{\mathrm{T}}$ e $\mathrm{DQO}_{\mathrm{F}}$ foi possível avaliar o desempenho dos reatores quanto a remoção de matéria orgânica. A série temporal da eficiência de remoção de $\mathrm{DQO}_{\mathrm{T}} \mathrm{e} \mathrm{DQO}_{\mathrm{F}}$ estão representadas na Figura 4.
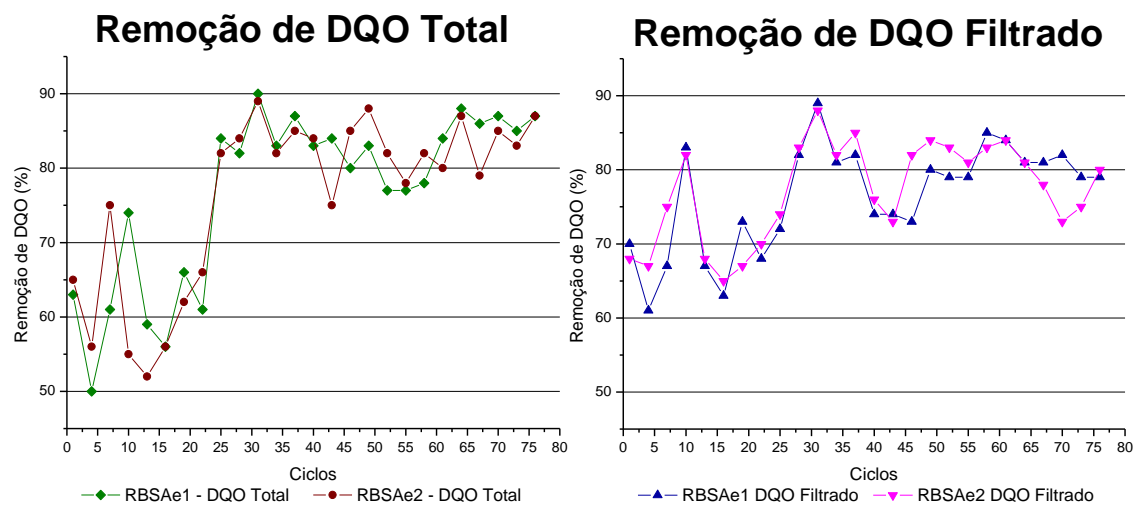

Figura 4 - Remoção de DQO Total e Filtrado nos reatores.

Avaliando os resultados da eficiência de remoção de $\mathrm{DQO}_{\mathrm{T}}$ e $\mathrm{DQO}_{\mathrm{F}}$ foi observado que a estabilização do sistema aconteceu no $25^{\circ}$ ciclo, vale ressaltar que a partida do reator foi realizada sem adição de inóculo, sendo que o lodo formado foi advindo do crescimento no próprio sistema. Cybis et al. (2004), realizaram o estudo com esgoto com característica fraco e obteve a estabilização do reator sequencial em batelada aeróbio após 4 dias de experimento (ciclos de 8 horas), porém, o seu sistema já havia sido inoculado com lodo de ETE e colocado em operação 48 dias antes do início do experimento.

A remoção de matéria orgânica em relação a $\mathrm{DQO}_{\mathrm{T}}$ foi em média de $84 \pm 4 \%$ no RBSAe1 e 83 $\pm 4 \%$ no RBSAe1. E em relação à $\mathrm{DQO}_{\mathrm{F}}$ a eficiência média em ambos os reatores foi de $80 \pm 4 \%$. Desses resultados, pode se observar que não houve diferença entre os dois reatores.

Os valores de remoção de sólidos estão representados nas Figuras 5. A partir do $25^{\circ}$ ciclo, onde foi considerado a estabilização dos reatores, os valores médios de eficiência de remoção de ST no RBSAe1 e RBSAe2 foi de $54 \pm 11 \%$ e $56 \pm 13 \%$, respectivamente. Podendo constatar que não houve diferença significativa entre os dois reatores. Assim como em relação a STV, onde o valor médio de eficiência de remoção foi de $70 \pm 17 \%$ para ambos. A eficiência de remoção SST foi em média de $87 \pm 10 \%$ no RBSAe1 e $86 \pm 16 \%$ no RBSAe2, para SSV obteve-se remoção média de 86 $\pm 10 \%$ no RBSAe1 e $84 \pm 19 \%$ no RBSAe2. 

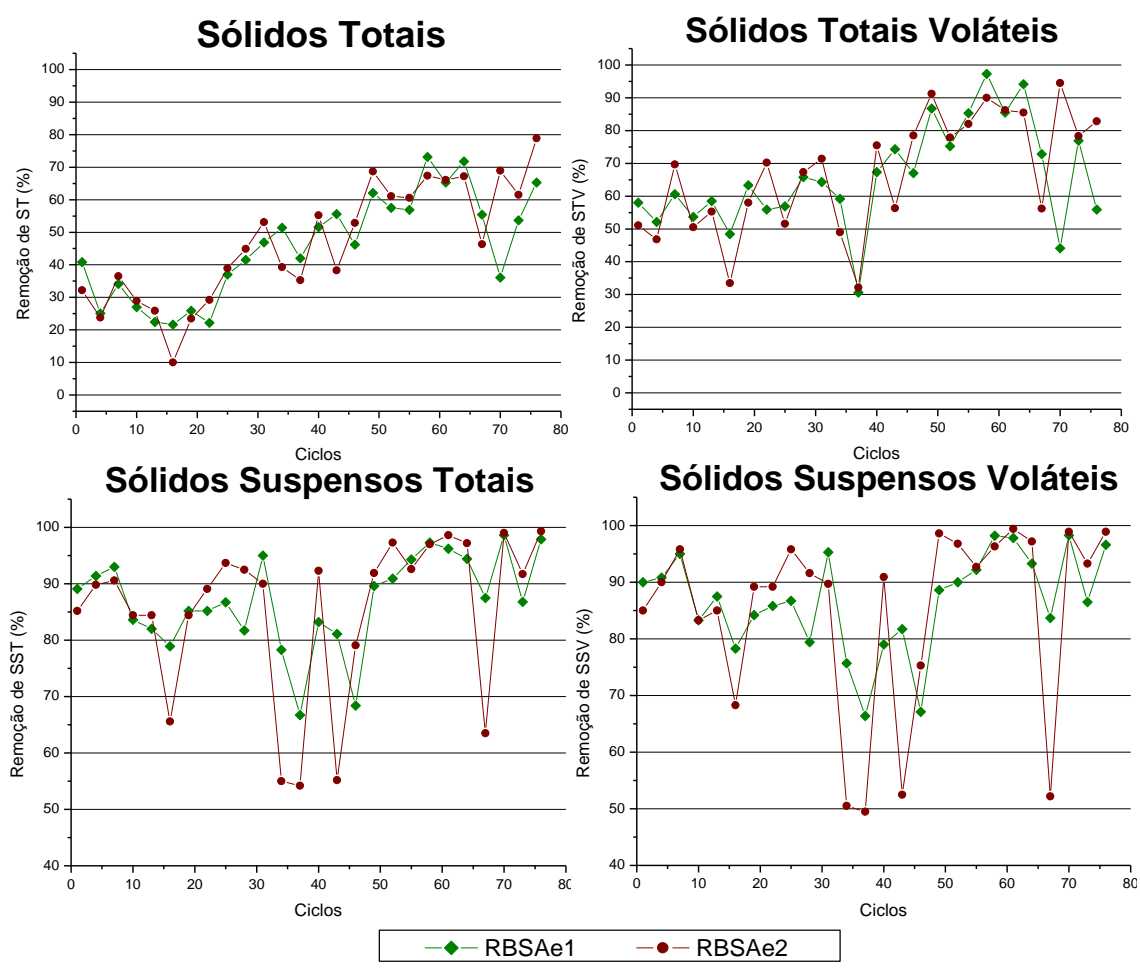

Figura 5 - Eficiência de remoção de ST, STV, SST e SSV nos reatores.

Dos resultados de DQO e sólidos pode se observar que não houve diferença significativa na eficiência de remoção destes parâmetros entre os reatores estudados. Fato este ressaltando que a quantidade de EMs da solução de EMs utilizada neste estudo não foi o suficiente para prover diferença da população no RBSAe1 comparado ao RBSAe2. Porém, mesmo não sendo possível observar um melhor desempenho no reator onde foi adicionado os EMs, os resultados mostraram que o sistema combinado o lodo ativado em Bateladas Sequenciais, e biomassa aderida apresentaram bons resultados, já que o RBSAe2, onde não houve a introdução de EMs, alcançou eficiência média de $83 \%$ de remoção de matéria orgânica como DQO Total.

\subsection{Análises microbiológicas}

Os resultados demonstraram que no esgoto bruto já haviam EMs presentes, sendo que a adição de EMs no início de cada ciclo não modificou de forma significativa a concentração da população desses micro-organimos no RBSAe1 (Tabela 1), mantendo todos na mesma ordem de grandeza do RBSAe2.

Os valores de concentração de EMs no esgoto sanitário encontrados no presente trabalho foram superiores aos encontrados por Maalin et al. (2009), que realizou análise em esgoto doméstico obtendo $1,7 \times 10^{6}$ para $\mathrm{BH}, 1 \times 10^{2}$ para leveduras e $1 \times 10^{4}$ para Lactobacilos (o presente trabalho foi considerado o grupo de bactérias fermentadoras de lactose), sendo que o autor não avaliou a quantidade de actinomicetos. Desse modo pode-se afirmar que é característico do esgoto sanitário possuir esses micro-organismos. 
Tabela 1 - Concentração (UFC/mL) de BH, leveduras, actinomicetos e BFL presente na solução EMs, esgoto afluente e total no sistema.

\begin{tabular}{ccccc|cccc}
\hline EMs & \multicolumn{4}{c|}{ RBSAe1 } & \multicolumn{4}{c}{ RBSAe2 } \\
\cline { 2 - 8 }$(\mathbf{U F C} / \mathbf{m L})$ & BH & LEV & ACT & BFL & BH & LEV & ACT & BFL \\
\hline $\begin{array}{c}\text { Adicionado } \\
\text { (UFC/mL) }\end{array}$ & $2,0 \times 10^{7}$ & $5,3 \times 10^{8}$ & $1,2 \times 10^{8}$ & $6,0 \times 10^{8}$ & - & - & - & - \\
$\begin{array}{c}\text { Esgoto Afluente } \\
(\text { UFC/mL) }\end{array}$ & $2,6 \times 10^{8}$ & $1,0 \times 10^{6}$ & $2,4 \times 10^{7}$ & $2,0 \times 10^{4}$ & $2,6 \times 10^{8}$ & $1,0 \times 10^{6}$ & $2,4 \times 10^{7}$ & $2,0 \times 10^{4}$ \\
$\quad$ Total & $2,6 \times 10^{8}$ & $1,5 \times 10^{6}$ & $2,4 \times 10^{7}$ & $6,2 \times 10^{4}$ & $2,6 \times 10^{8}$ & $1,0 \times 10^{6}$ & $2,4 \times 10^{7}$ & $2,0 \times 10^{4}$ \\
\hline
\end{tabular}

Os resultados das análises microbiológicas do lodo e na espuma no $28^{\circ}$ e $76^{\circ}$ ciclo estão descritas na Tabela 2.

Tabela 2 - Quantidade de EMs e BH presentes no lodo e aderidas à espuma.

\begin{tabular}{|c|c|c|c|c|c|c|c|c|}
\hline & \multicolumn{4}{|c|}{ Lodo (UFC/mL) } & \multicolumn{4}{|c|}{ Espuma (UFC/Espuma) } \\
\hline & \multicolumn{2}{|c|}{$28^{\circ}$ ciclo } & \multicolumn{2}{|c|}{$76^{\circ}$ ciclo } & \multicolumn{2}{|c|}{$28^{\circ}$ ciclo } & \multicolumn{2}{|c|}{$76^{\circ}$ ciclo } \\
\hline & RBSAe1 & RBSAe2 & RBSAe1 & RBSAe2 & RBSAe1 & RBSAe2 & RBSAe1 & RBSAe2 \\
\hline BH & $7,0 \times 10^{7}$ & $6,4 \times 10^{7}$ & $3,5 \times 10^{6}$ & $2,5 \times 10^{7}$ & $1,6 \times 10^{10}$ & $1,4 \times 10^{10}$ & $1,0 \times 10^{10}$ & $2,5 \times 10^{10}$ \\
\hline ACT & $4,0 \times 10^{6}$ & $3,8 \times 10^{7}$ & $6,0 \times 10^{6}$ & $1,5 \times 10^{7}$ & $9,0 \times 10^{8}$ & $4,3 \times 10^{8}$ & $3,1 \times 10^{8}$ & $1,1 \times 10^{9}$ \\
\hline LEV & $9,7 \times 10^{5}$ & $5,5 \times 10^{5}$ & $4,0 \times 10^{5}$ & $6,3 \times 10^{5}$ & $3,0 \times 10^{7}$ & $4,0 \times 10^{7}$ & $3,5 \times 10^{7}$ & $3,05 \times 10^{8}$ \\
\hline BFL & $9,7 \times 10^{5}$ & $5,5 \times 10^{5}$ & $4,0 \times 10^{3}$ & $1,7 \times 10^{4}$ & $3,5 \times 10^{6}$ & $1,9 \times 10^{5}$ & $1,5 \times 10^{6}$ & $1,6 \times 10^{5}$ \\
\hline
\end{tabular}

Por meio dos dados da Tabela 2, é possível observar que havia biomassa de BH e EMs presentes no lodo e aderidas à espuma, sendo que os maiores valores de concentrações nos reatores foi de BH e ACT nos dois ciclos analisados, mantendo assim a mesma proporção encontrada na caracterização inicial dos reatores. Destes resultados é possível afirmar que a biomassa de EMs está presente no sistema na forma suspensa (lodo) e também são capazes de formar biofilme (aderidas à espuma), verificando que os EMs são capazes de se desenvolver em esgoto sanitário tornando estes micro-organismos um potencial agente biológico consumidor da matéria orgânica presente em esgoto.

\section{CONCLUSÃO}

A concentração de BFL, ACT e LEV presente na solução de EMs utilizado neste trabalho não foi o suficiente para prover diferença significativa no desempenho dos reatores durante o período de monitoramento. Porém, mesmo não sendo possível avaliar a diferença de desempenho foi possível concluir que o sistema utilizado apresentou bom desempenho quanto a remoção de DQO e sólidos.

Também foi possível concluir que os EMs são capazes de se desenvolver em esgoto sanitário 
tornando estes microrganismos um potencial agente biológico consumidor da matéria orgânica presente em esgoto sanitário.

\section{REFERÊNCIAS}

AGUILAR, R. M. T. Efecto de los Microorganismos Eficientes (ME) em las aguas residuales de La granja porcina de Zamorano, Honduras. 2012. 22 f. Projeto especial de graduação apresentado como requisito parcial para optar o titulo de Engenheiro Agrônomo em grau acadêmico de Licenciatura. Zamorano Escola Agrícola Pan-americano, Zamorano, 2012.

APHA; AWWA; WEF. Standard Methods For the Examination of Water and Wastewater. 22 ed. Washington, DC: APHA, 2012.

AYGUN, A.; NAS, B.; BERKTAY, A. Influence of high organic loading rates on COD removal and sludge production in moving bed biofilm reactor. Environmental Engineerging Science. Vol. 25. 2008.

BALASUBRAMANIAN, N.; MUTHUKUMAR, M. Performace of HUASB reactor for treating paper \& Pulp wastewater using Effective Microorganism (EM). Internacional Journal of Engineering Science and Technology (IJEST). Vol 4. N. 6., p. 2453-2461, 2012.

BENERJI, S.; WOOD, M.; FARRELLY, P. Evaluation of Effective Microorganisms Wastewater Treatment Method for Use in a Solar Aquatic Facility in Bozeman, Montana, USA. 2002. Disponível em: <http://envismadrasuniv.org/pdf/Waste\%20Water\%20Treatment.pdf>. Acesso em: 05 de junho de 2013.

BONFIM, F. P. G.; HONÓRIO, I. C. G.; REIS. I. L.; PEREIRA, A. J.; SOUZA. D. B. Caderno dos Microrganismos Eficientes (EM): Instruções práticas sobre o uso ecológico e social do EM. 2. ed. Viçosa: 2011.

CYBIS, L. F. A.; SANTOS, A. V.; GEHLING, G. R. Eficiência do reator sequencial em batelada (RSB) na remoção de nitrogênio no tratamento de esgoto doméstico com DQO baixa. Eng. sanit. ambient. Vol. 9. n. 3. p. 260-264. Jul/set, 2004.

MAALIN, M. K.; MGANA, S. M.; KASSENGA, G. R.; MOHAMMED, S.M. Role of effective microorganisms on the reduction of coliform in the Upflow Anaerobic Sludge Blanket Reator treating domestic wastewater under tropical conditions. Proceedings of the 11th International Conference on Environmental Science and Technology. Chania: 2009. P. B568-B574.

NURUL, Z. R. A.; HASSAN, M.A.A.; MAHMOOD, I.; KHAMIS, A.K. Treatment of Rubber Processing Wastewater by Effective Microorganisms Using Anaerobic Sequencing Batch Reactor. $J$. Agrobiotech. Vol. 4, p. 1-15, 2013. 